Nephrologe 2020 · 15:210-215

https://doi.org/10.1007/s11560-020-00444-4

Online publiziert: 26. Juni 2020

(c) Springer Medizin Verlag $\mathrm{GmbH}$, ein Teil von

Springer Nature 2020

\section{Redaktion}

T. Feldkamp, Kiel

O. Witzke, Essen

Im Dezember 2019 wurde in der Stadt Wuhan, der Hauptstadt der Provinz Hubei in Zentralchina, eine Häufung unerklärlicher Lungenentzündungen gemeldet. Im Januar 2020 wurde der Erreger dieser bilateralen interstitiellen Lungenentzündung identifiziert: das neue Coronavirus SARS-CoV-2 (,severe acute respiratory syndrome coronavirus $\left.2^{\prime \prime}\right)$, das zum $\beta$-Coronavirus-Cluster gehört, zu dem auch die SARS und MERS („Middle East respiratory syndrome") verursachenden Viren gehören [1]. Ende Januar wurden bereits 282 bestätigte Fälle gemeldet. Nachfolgend verbreitete sich die als COVID-19 („coronavirus disease 2019“) bekannt gewordene Erkrankung innerhalb weniger Wochen in nahezu alle Länder der Welt. Nach Angaben der Weltgesundheitsorganisation(WHO) erreichte die SARS-CoV-2-Infektion Mitte März2020 pandemische Ausmaße. In Europa wurden dabei Italien und Spanien besonders hart getroffen. In Deutschland wurde die erste größere Zahl von Infizierten im Februar nach einer Karnevalsveranstaltung in der Gemeinde Gangelt gemeldet. Soziale Kontakteinschränkungen und ein „Lockdown“ waren die Folgen.

Das klinische Spektrum einer Infektion mit SARS-CoV-2 reicht vom symptomlosen Verlauf bis zu schweren respiratorischen Symptomen mit Beatmungspflicht und erhöhter Sterblichkeit. Bei etwa $80 \%$ der Patienten ist der Verlauf milde, und es kommt zu einer spontanen Erholung. Etwa $15 \%$ der Patienten entwickeln eine schwer verlaufende COVID19-Erkrankung, und 5\% der Patienten sind kritisch krank [2].

\author{
H. Rohn' ${ }^{1}$ T. Feldkamp ${ }^{2} \cdot$ O. Witzke' \\ ${ }^{1}$ Klinik für Infektiologie, Westdeutsches Zentrum für Infektiologie (WZI), Universitätsmedizin Essen, \\ Universität Duisburg-Essen, Essen, Deutschland \\ ${ }^{2}$ Klinik für Nieren- und Hochdruckkrankheiten, Innere Medizin IV, Universitätsklinikum Schleswig- \\ Holstein, Campus Kiel, Kiel, Deutschland
}

\title{
COVID-19 und die Niere
}

Die Symptome der COVID-19-Erkrankung ähneln denen der gewöhnlichen saisonalen Grippe. Bei fast allen Patienten sind Fieber und trockener Husten die beiden am häufigsten berichteten Krankheitszeichen. 20\% der Patienten klagen über Dyspnoe. „Klassische“Erkältungssymptome, Halsschmerzen sowie eine verstopfte oder laufende Nase treten nur in etwa $5-10 \%$ der erfassten Fälle auf. Ungefähr 5-10\% der Patienten zeigen primär gastrointestinale Syndrome mit Durchfall, Übelkeit und z.T. auch Bauchschmerzen und Erbrechen [2].

\section{》) COVID-19 stellt keine \\ homogene, eine ganze \\ Bevölkerung einheitlich \\ betreffende Epidemie dar}

COVID-19 stellt keine homogene, eine ganze Bevölkerung einheitlich betreffende Epidemie dar. V.a. ältere Menschen und Patienten mit Vorerkrankungen wie Herz-Kreislauf-Erkrankungen, Diabetes, chronischen Atemwegserkrankungen, malignen Erkrankungen und mit geschwächtem Immunsystem haben ein höheres Risiko, einen schweren COVID-19-Verlauf zu entwickeln [3]. Männer haben ein höheres Risiko für einen schweren Verlauf als Frauen. Ob die Geschlechterunterschiede auf Lebensstilfaktoren oder biologische Unterschiede zurückzuführen sind, ist aktuell unklar. Schwere Verläufe bei Kindern und Jugendlichen sind extrem selten. Jedoch häufen sich Fallberichte, die eine SARS-CoV-2-Infektion bei Kindern und Jugendlichen mit einer Kawasaki-
Syndrom-ähnlichen multisystemischen Inflammation in Verbindung bringen.

Die Virusübertragung erfolgt von Mensch zu Mensch durch Inhalation von Tröpfchen oder Aerosolen. Der längere Aufenthalt in schlecht belüfteten Räumen erhöht die Wahrscheinlichkeit einer Übertragung, wohingegen eine Übertragung im Freien (bei Wahrung des Mindestabstands) sehr selten vorkommt. Die mediane Inkubationszeit von Exposition bis zum Auftreten erster Symptome beträgt 4 Tage, die maximale Inkubationszeit wird mit 14 Tagen angenommen. Das Virus kann bereits 1 bis 2 Tage vor Symptombeginn von asymptomatischen Trägern ausgeschieden werden.

\section{Diagnostik}

Der Nachweis von SARS-CoV-2 erfolgt vorzugsweise aus einem tiefen Nasenrachenabstrich mittels PCR („polymerase chain reaction"). Dabei ist auf eine ausreichende Tiefe des Abstrichs zu achten, um falsch-negative Testergebnisse $\mathrm{zu}$ vermeiden. Auch bei korrekter Abstrichentnahme können falsch-negative Testergebnisse auftreten. Bei klinischem Verdacht sollten daher zwingend serielle Testungen erfolgen. Im späteren Verlauf der Erkrankung (Pneumonie, ARDS [„acute respiratory distress syndrome“]) kann der Rachenabstrich bereits wieder negativ sein, während noch infektiöse Viruslast in den unteren Atemwegen nachweisbar ist $[4,5]$. Bei diesen Patienten muss Material aus den unteren Atemwegen (Sputum, Trachealsekret, bronchoalveoläre Lavage) untersucht werden. 

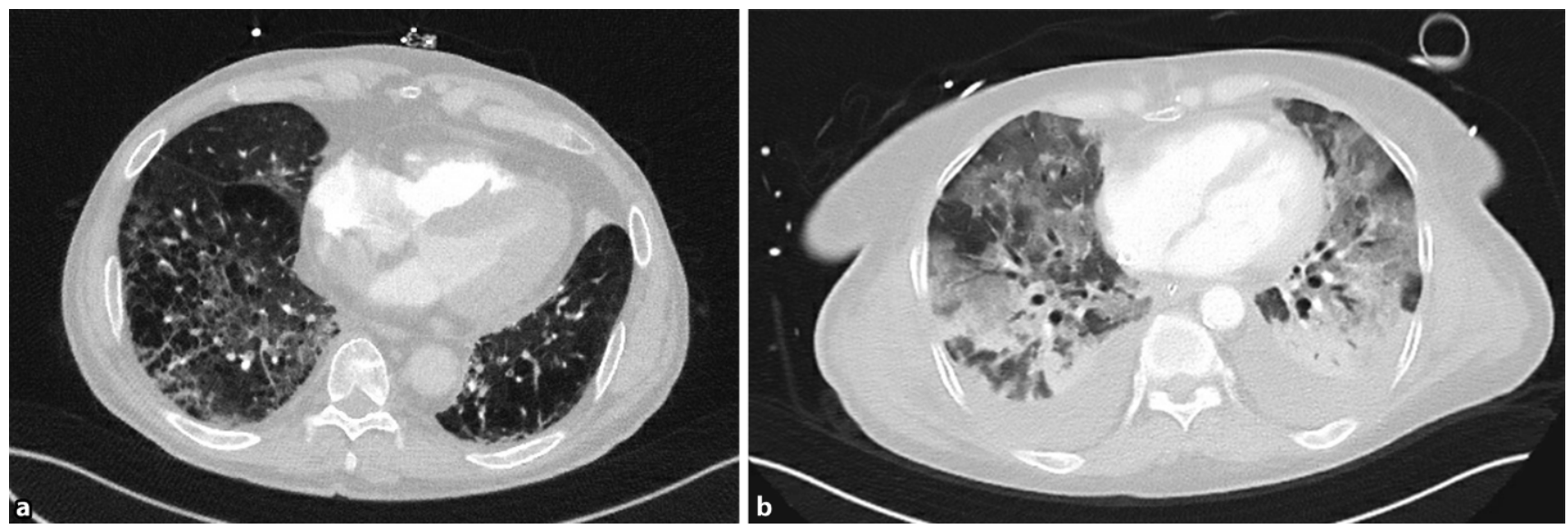

Abb. 1 ॥ CT(Computertomographie)-ThoraxUntersuchungen von 2Patienten mitgesicherter, schwererCOVID-19("coronavirus disease 2019")-Pneumonie: a flaue, bipulmonale Milchglasinfiltrate bei panlobuärem Lungenemphysem; $\mathbf{b}$ bipulmonale Milchglasinfiltrate mit oberlappenbetonter interlobulärer Septenverdickung sowie flächigen dorsobasalen Konsolidierungen und beidseitigen Pleuraergüssen

Kompakte Testsysteme als Point-ofCare-Diagnostik analog dem Kartuschentest zum Nachweis von Influenzaviren oder des humanen respiratorischen Synzytialvirus (RSV) sind zunehmend verfügbar. Diese Testsysteme liefern bereits Ergebnisse in weniger als einer Stunde und eignen sich somit v.a. für Notaufnahmen.

Auch Antikörpertests (zumeist ELISA[,enzyme-linked immunosorbent assay"]-basiert) sind mittlerweile verfügbar, die Antikörper der Klasse IgA (Immunglobulin A), IgM und IgG, die gegen Strukturproteine von SARS-CoV-2 gerichtet sind, nachweisen können [6]. Die Antikörpertests sind nur sehr eingeschränkt zum Nachweis einer akuten Infektion geeignet. Problematisch ist auch die je nach Test vorhandene Kreuzreaktivität gegenüber endemisch zirkulierenden Coronaviren [7]. Erste Studien zu neutralisierenden Antikörpern, die auf eine protektive Immunität hindeuten, zeigen, dass diese am Ende der zweiten Woche nach Symptombeginn nachweisbar sind [8]. Unklar ist, wie regelhaft und dauerhaft dieser Immunstatus aufgebaut wird. Die Erfahrungen mit MERS und SARS lassen darauf schließen, dass die Immunität bis zu 3 Jahre anhalten könnte $[9,10]$.

\section{Labor}

Laborchemisch zeigt sich häufig (ca. $80 \%$ ) eine Lymphopenie, bei einem Drittel der
Patienten eine Leukopenie. Die meisten Patienten haben einen normalen Procalcitoninwert. Bei deutlich erhöhten Werten muss an eine bakterielle Superinfektion gedacht werden. Das C-reaktive Protein (CRP) ist sehr häufig erhöht, und hohe Werte scheinen mit einer schlechteren Prognose zu korrelieren [11]. Eine Thrombozytopenie, LDH(Laktatdehydrogenase)-Wert-Erhöhung oder D-Dimer-Wert-Erhöhung findet sich bei etwa $40 \%$ der Patienten.

\section{》) Erhöhte D-Dimer-Werte sind als Warnsignal für einen schweren Verlauf zu werten}

V.a. erhöhte D-Dimer-Werte sind als Warnsignal für einen schweren Verlauf zu werten. Zahlreiche Studien belegen inzwischen, dass eine COVID-19Erkrankung mit einem erhöhten Thrombose- und Embolierisiko einhergeht. Das Auftreten dieser Gerinnungsstörungen ist prognoseentscheidend in Bezug auf die Morbidität und Mortalität bei COVID-19-Patienten.

Laut aktueller Empfehlungen der Fachgesellschaften sollte bei COVID19-Patienten das individuelle Thromboserisiko evaluiert und die Indikation zur prophylaktischen Antikoagulation großzügig gestellt werden. Angepasst an das individuelle Thromboserisiko und den D-Dimer-Wert, sind auch höhere Dosen an Antikoagulanzien möglich, zumal schwere Blutungen bei COVID19 bisher nicht beschrieben wurden. Niedermolekulare Heparine haben neben der Gerinnungshemmung auch noch nicht gerinnungshemmende Eigenschaften, wie etwa eine verringerte Interleukin(IL)-6-Freisetzung, was bei COVID-19-Patienten sogar von Vorteil sein könnte.

\section{Bildgebung}

Das konventionelle Röntgenthoraxbild zeigt bei $50-60 \%$ der COVID-19-Erkrankten Veränderungen auf, die Computertomographie(CT)-Untersuchung der Lunge in etwa $85 \%$ der Fälle. Sichtbar werden milchglasartige Infiltrate, bilaterale oder seltener unilaterale Verdichtungen und/oder interstitielle Zeichnungsvermehrung, später dominante Crazy-paving-Muster (siehe - Abb. 1). Damit sind die bildgebenden Merkmale der COVID-19-Pneumonie in der CT unspezifisch. Identische Muster können auch bei den meisten anderen viralen oder atypischen Pneumonien auftreten, verursacht z. B durch Influenza, Zytomegalievirus (CMV), Herpes-simplexVirus (HSV) oder Pneumocystis jirovecii. Eine Koinfektion v.a. mit Mycoplasma pneumoniae und Aspergillus spp. findet sich bei bis zu $40 \%$ der COVID19-Patienten [11]. Die CT-Thorax-Untersuchung ermöglicht es, pulmonale Begleitbefunde $\mathrm{zu}$ erheben und den Schweregrad der COVID-19-Pneumo- 
nie abzuschätzen. Zusammen mit den entsprechenden Laborparametern können so Patienten mit hohem Risiko für einen schweren Verlauf frühzeitig identifiziert werden. Für das alleinige Screening auf COVID-19 ist die CTUntersuchung nicht empfohlen.

Symptome der Atemwege und der Lunge stehen bei der COVID-19-Erkrankung im Vordergrund, doch SARSCoV-2 kann auch die Nieren angreifen.

\section{Akutes Nierenversagen als Folge der SARS-CoV-2-Infektion}

Die Nieren sind neben der Lunge ein entscheidendes Zielorgan von SARSCoV-2. Die Nierenbeteiligung bei COVID-19 kann sich als akutes Nierenversagen (ANV), Proteinurie oder Hämaturie bemerkbar machen und ist mit einer deutlich erhöhten Sterblichkeit assoziiert [12].

\section{》) Die Nieren sind neben der Lunge ein entscheidendes Zielorgan von SARS-CoV-2}

In Beobachtungsstudien entwickeln 5-36,6\% der hospitalisierten COVID19-Patienten ein ANV unterschiedlichen Schweregrads. In einer multizentrischen Kohortenstudie aus den USA lag der Anteil der Patienten mit dialysepflichtigem ANV bei 5\% [13]. Dabei ist der Anteil an ANV bei beatmungspflichtigen Patienten mit fast $90 \%$ signifikant höher als bei nichtbeatmeten Patienten (ca. 21\%; [12]). Laut einer aktuellen Umfrage der Deutschen Gesellschaft für Nephrologie (DGfN) im April 2020, an der 52 nephrologische Kliniken teilnahmen, wurden 504 COVID-19-Patienten auf Intensivstationen behandelt, von denen 161 ein dialysepflichtiges ANV hatten $(32 \%)$.

Obduktionsstudien konnten zeigen, dass die menschliche Niere ein spezifisches Ziel für eine SARS-CoV-2Infektion darstellt [14-16]. SARS-CoV2-Antigene akkumulieren in den Nierentubuli. Eine SARS-CoV-2-Infektion kann neben der Lunge und der Niere auch das Endothel, das Herz und das Hirn schädigen [17]. Morphologisch imponiert eine prominente Endothelitis der Blutgefäße. Dies könnte erklären, wie SARS-CoV-2 über eine generalisierte Endothelentzündung und damit verbundene Gefäßverengung mit reduzierter Blutversorgung des betroffenen Organs ein tödliches Organversagen auslöst.

CT-Untersuchungen der Nieren zeigten zudem eine verminderte Dichte des Nierengewebes, was indirekt auf eine Entzündung und ein Ödem hindeutet.

$\mathrm{Ob}$ das Zytokinfreisetzungssyndrom auch ein Merkmal der SARS-CoV-2Infektion ist und wie bei SARS zum ANV beiträgt, ist noch nicht bekannt. Jedoch scheinen Tocilizumab und Sarilumab, beides spezifische Inhibitoren von IL-6, einem Hauptfaktor bei der Entstehung des Zytokinfreisetzungssyndroms, bei schweren COVID-19-Fällen vorteilhaft für den Krankheitsverlauf zu sein. Tocilizumab wird aktuell in einer randomisierten, kontrollierten Studie an COVID-19-Patienten untersucht.

Es bleibt zu klären, ob das ANV weitgehend auf hämodynamische Veränderungen und die Zytokinfreisetzung zurückzuführen ist oder ob das Virus selbst zu einer direkten Zytotoxizität führt.

Der hohe Nierentropismus von SARSCoV-2 kann z. T. durch die Affinität des Virus für membrangebundenes ACE2 (,angiotensin-converting enzyme 2“) erklärt werden [18]. SARS-CoV-2 nutzt ACE2 als „Türöffner“, um Zugang zu seinen Zielzellen zu erhalten $[19,20]$. ACE2 wird v.a. in den Alveolarzellen in der Lunge exprimiert, aber auch in anderen Organen, darunter Niere, Herz, Darm und Mund-Rachen-Raum (was den Eintritt des Virus in den Wirt erleichtert; [21]).

\section{ACE-Hemmer und SARS-CoV-2}

Aus den bisherigen epidemiologischen Daten geht hervor, dass über zwei Drittel der Patienten, die an COVID-19 starben, an Diabetes oder Herz-KreislaufErkrankungen litten [22]. Die meisten dieser Patienten erhielten eine leitliniengerechte blutdrucksenkende Therapie mit ACE-Hemmern oder Angiotensinrezeptorblockern (ARB). Diese beiden Medikamentengruppen sind in der Lage, die ACE2-Expression in den Nieren und im
Nephrologe 2020 15:210-215 https://doi.org/10.1007/s11560-020-00444-4 (c) Springer Medizin Verlag GmbH, ein Teil von Springer Nature 2020

\section{H. Rohn · T. Feldkamp • O. Witzke \\ COVID-19 und die Niere}

\section{Zusammenfassung}

Welche drastischen Folgen neue Infektionskrankheiten für Mensch und Gesellschaft haben können, wird aktuell an COVID-19 („,coronavirus disease 2019“) deutlich. Seit seiner Erstbeschreibung im Dezember 2019 beherrscht SARSCoV-2 (,severe acute respiratory syndrome coronavirus $2^{\prime \prime}$ ) das aktuelle wissenschaftliche und öffentliche Interesse.

Schlüsselwörter

SARS-Coronavirus-2 - Pandemien .

Akutes Nierenversagen · Chronische

Nierenerkrankung · COVID-19

\section{COVID-19 and the kidneys}

\section{Abstract}

The drastic consequences that emerging infectious diseases can have for people and society are currently being demonstrated by coronavirus disease 2019 (COVID-19). Since its initial description in December 2019 severe acute respiratory syndrome coronavirus 2 (SARS-CoV-2) has dominated current scientific and public interest.

Keywords

SARS-Coronavirus-2 - Pandemics - Acute kidney injury - Chronic renal insufficiency . COVID-19

Herzen um das 2- bis 5-Fache zu erhöhen [23]. Konsequenterweise kam schnell die Diskussion auf, ob der Einsatz dieser Medikamente Patienten anfälliger für eine SARS-CoV-2-Infektion macht und für schwerer verlaufende COVID-19-Erkrankungen prädisponiert [24].

Nach derzeitigem Wissensstand gibt es keine Belege für einen Zusammenhang zwischen der Einnahme von ACE-Hemmern bzw. ARB und einem schweren COVID-19-Verlauf. Einige große Studien und eine systematische Übersicht weisen auf keinen Zusammenhang zwischen der Einnahme dieser Wirkstoffe und dem Schweregrad von COVID-19 hin [25-29], während andere Arbeiten sogar darauf hindeuten, dass ACE-Hem- 
mer den Schweregrad der Erkrankung mildern könnten [30-32]. Dies wird auch von tierexperimentellen Studien gestützt, die in der Summe zeigen, dass eine erhöhte ACE2-Expression während einer Coronavirusinfektion protektiv wirkt.

Die offiziellen Stellungnahmen der European Society of Hypertension (ESH) und der European Society of Cardiology (ESC) empfehlen daher, dass die Blutdruckmedikamente bei Patienten mit einem Risiko für COVID-19 oder bei Patienten, bei denen bereits COVID-19 diagnostiziert wurde, nach Möglichkeit nicht verändert werden sollten.

\section{Chronische Nierenerkrankung und SARS-CoV-2}

Obwohl die verfügbaren epidemiologischen Daten bestätigt haben, dass das ANV einer der Hauptrisikofaktoren für die Prognose einer COVID-19-Erkrankung ist, sind die möglichen Auswirkungen von COVID-19 auf Patienten, die von anderen Nierenerkrankungen betroffen sind, in dieser Phase der Pandemie noch unklar. V.a. Patienten mit terminaler Nierenerkrankung sind aufgrund des oft höheren Alters und der Häufigkeit von Komorbiditäten wie Diabetes und Bluthochdruck anfällig für eine schwer verlaufende COVID-19-Erkrankung [3].

Angesichts von COVID-19 werden v. a. Dialysezentren vor besondere Herausforderungen gestellt. Als Hilfestellung haben die nationalen und internationalen Fachgesellschaften vorläufige Richtlinien herausgegeben [33].

Was wir bisher über den Verlauf von COVID-19 bei Patienten an der Hämodialyse (HD) wissen, geht auf Untersuchungen zurück, die sich mit dem Verlauf des Krankheitsausbruchs in einem einzigen HD-Zentrum im Renmin-Krankenhaus der Universität Wuhan beschäftigt haben. In diesem Zentrum gab es insgesamt 37 Fälle von COVID-19 bei 230 HDPatienten und 4 Fälle bei 33 Mitarbeitern. Bei den meisten Patienten waren die COVID-19-Symptome mild, und es mussten keine Patienten intensivmedizinisch versorgt werden. Während der Epidemie starben 7 HD-Patienten, darunter 6 mit COVID-19-Erkrankung. Die
Todesursachen standen offenbar nicht im Zusammenhang mit einer Lungenentzündung. Die Analyse von peripheren Blutproben deran COVID-19 erkrankten HD-Patienten zeigte eine bemerkenswerte Verringerung der Anzahlvon T-Zellen, T-Helfer-Zellen, Killer-T-Zellen und natürlichen Killerzellen (NK-Zellen) sowie niedrigere Serumspiegel von proinflammatorischen Zytokinen im Vergleich zu Nicht-HD-Patienten mit COVID-19.Zusammenfassend berichtete diese Studie, dass HD-Patienten mit COVID-19 eine leichte Erkrankung erleiden, die sich nicht zu einer ausgewachsenen Lungenentzündung entwickelt, was wahrscheinlich auf die reduzierte Funktion des Immunsystems und die geringere Zytokinfreisetzung zurückzuführen ist. Dieser Bericht stellt jedoch auch dar, dass HDPatienten einem hohen Risiko ausgesetzt sind, sich mit SARS-CoV-2 zu infizieren, und dass zusätzliche Präventionsmaßnahmen für das Management der Epidemie in HD-Zentren unerlässlich sind.

Im Hinblick auf COVID-19 und die Patienten unter immunsuppressiver Therapie bei nephrologischen Erkrankungen gibt es wenige Daten. Analog zu anderen Infektionskrankheiten ist aber davon auszugehen, dass sowohl nierentransplantierte Patienten als auch Patienten unter immunsuppressiver Medikation aufgrund von Autoimmunerkrankungen ein höheres Risiko für einen schweren COVID-19-Verlauf haben. Als behandelnder Arzt wird man zwangsläufig vor die Frage gestellt, ob eine immunsuppressive Therapie initiiert bzw. fortgesetzt werden kann oder, falls möglich, besser verschoben wird. Dabei ist immer das Risiko einer verstärkten Immunsuppression oder Hyperinflammation gegenüber der Prognoseverschlechterung der zugrunde liegenden Erkrankung abzuwägen. Wirkliche Evidenz gibt es bisher noch nicht. Die aktuellen Empfehlungen basieren auf Experteneinschätzungen der Fachgesellschaften und der hoffentlich raschen Verarbeitungen von Evidenzen aus Registerstudien wie beispielsweise den europäischen Registern ERACODE (The ERAEDTA COVID-19 Database For Patients on Dialysis Or Living With A Kidney Transplant) und LEOSS (Lean European Open Survey on SARS-CoV-2 Infected
Patients). In letzterem waren Ende Mai 202051 transplantierte SARS-CoV-2-infizierte Patienten registriert, 27 davon waren nieren- und 4 Patienten nieren- und pankreastransplantiert. $85 \%$ der transplantierten Patienten aus dem LEOSSRegister waren länger als 1 Jahr transplantiert. Jeder vierte Patient hatte einen kritischen Verlauf und jeder zehnte verstarb. Diese Fallmortalität liegt damit deutlich höher als die COVID-19-Mortalität in der Normalbevölkerung.

\section{》) Transplantierte Personen sind als Hochrisikogruppe besonders zu schützen}

Es ist mit Nachdruck zu betonen, dass transplantierte Personen keinen vermeidbaren Infektionsrisiken auszusetzen und als Hochrisikogruppe besonders zu schützen sind.

Aus In-vitro-Studien zu MERS-CoV und SARS-CoV ist bekannt, dass sowohl Cyclosporin als auch Mycophenolat-Mofetil (MMF) die virale Replikation hemmen [34]. Zwar gibt es keinen aktuellen Bericht über die Wirkung dieser Immunsuppressiva auf SARS-CoV-2, ERA (European Renal Association) und EDTA (European Dialysis and Transplant Association) empfehlen aber dennoch, bei ambulanten, klinisch stabilen Patienten ohne Infektionszeichen die bestehende immunsuppressive Therapie nicht zu verändern. - Tab. 1 fasst die aktuellen ERA-EDAT-Empfehlungen bezüglich der Umstellung der immunsuppressiven Therapie bei Patienten mit unterschiedlichen Schweregraden einer COVID-19-Erkrankung zusammen.

Die Datenlage zu hoch dosierten Steroiden ist uneinheitlich. Diese sollten daher bei ARDS in keinem Fall routinemäßig eingesetzt werden. Höhere Dosierungen scheinen die Virämie zu verlängern und das Pilzwachstum zu fördern und werden aufgrund dieser nachteiligen Effekte im Augenblick nicht empfohlen.

\section{Potenzielle Nierenspender und SARS-CoV-2}

Wie oben bereits beschrieben, haben Obduktionsstudien gezeigt, dass SARS- 
Tab. 1 Die aktuellen ERA(European Renal Association)-EDTA(European Dialysis and Transplant Association)-Empfehlungen bezüglich der Umstellung der immunsuppressiven Therapie bei Patienten mit unterschiedlichen Schweregraden einer COVID-19(,'coronavirus disease 2019“)-Erkrankung

Asymptomatische, stabile Patienten ohne Anzeichen für eine SARS-CoV-2-Infektion

Empfehlung Keine Veränderung der bestehenden immunsuppressiven Therapie

Patienten mit leichter COVID-19-Erkrankung ohne klinischen oder radiologischen Anhalt für eine COVID-19-Pneumonie

Empfehlung

Falls der Patient eine Dreifachtherapie erhält: STOP MPA/AZA/mTOR, weiter mit einer CNI-Steroid-Erhaltungstherapie

Falls der Patient eine duale Therapie erhält: Fortsetzung der dualen Therapie Falls die duale Therapie steroidfrei ist: bei CNI + MPA/mTOR erwägen, MPA/ mTOR durch niedrig dosierte Steroide zu ersetzen

Bei MPA + mTOR den Ersatz von MPA oder mTOR durch niedrig dosierte Steroide erwägen

Eine Reduktion der CNI-Dosis (auf die untere Grenze des therapeutischen Bereichs entsprechend dem immunologischen Risiko) ist zu erwägen, wenn in den ersten 3 bis 5 Tagen keine deutliche Besserung eintritt

Patienten mit Anzeichen einer leichten COVID-19-Pneumonie (Sauerstoffsättigung: 94-95\% unter Raumluft, AF: 25-29/min; oder verdächtige Befunde im Röntgen- oder (T-Thorax)

Empfehlung Hochrisikopatienten aufgrund des Alters über 70 Jahre oder aufgrund von Komorbiditäten bzw. Risikofaktoren (Diabetes, Herz- oder Lungenerkrankung, starkes Rauchen, BMI $>30 \mathrm{~kg} / \mathrm{m}^{2}$, eGFR $<30 \mathrm{ml} / \mathrm{min} / 1,73 \mathrm{~m}^{2}$, Lymphozytendepletionstherapie innerhalb der letzten 3 bis 6 Monate):

STOP MPA/AZA/mTOR, STOP CNI, Erhöhung (oder Beginn) einer Steroidtherapie mit $15-25 \mathrm{mg} / \mathrm{Tag}$

Keine Hochrisikopatienten (wie oben definiert):

STOP MPA/AZA/mTOR, Fortführung einer dualen CNI-Steroidtherapie; CNI-

Zielspiegel reduzieren: CsA $50 \pm 15 \mathrm{ng} / \mathrm{ml}$, TAC: $3 \pm 1 \mathrm{ng} / \mathrm{ml}$, Steroidtherapie in der Erhaltungsdosis fortführen

Bei Patienten, die mit einer antiretroviralen Therapie beginnen, müssen CNI abgesetzt und das im letzten Abschnitt beschriebene Monitoring durchgeführt werden

Patienten mit einer schweren COVID-19-Pneumonie (Sauerstoffsättigung $<94 \%$ unter Raumluft, AF >30/min; instabiler oder sich verschlechternder Verlauf oder Notwendigkeit einer nicht-invasiven Beatmung bzw. Überwachung auf einer Intensivstation mit oder ohne invasive Beatmung)

Empfehlung Alle Immunsuppressiva absetzen, Steroiddosis auf 15-25 mg/Tag erhöhen/ beginnen

Bei Patienten mit hohem Risiko für eine Abstoßung sollte die Fortsetzung einer niedrig dosierten $\mathrm{CNI}$-Therapie erwogen werden

Medikamenteninteraktion zwischen Chloroquin, antiviralen Medikamenten und der Immunsuppression

Empfehlung

www.covid-19-druginteractions.org

Patienten, die eine antiretrovirale Medikation beginnen, die Ritonavir oder Cobicistat enthält, müssen mTOR-Inhibitoren und CNI absetzen

SARS-CoV-2 "severe acute respiratory syndrome coronavirus 2", MPA Mycophenolsäure, AZA Azathioprin, $m T O R$ "mechanistic target of rapamycin", CNI Calcineurininhibitor, AF Atemfrequenz, CT Computertomographie, BMI Body-Mass-Index, eGFR geschätzte glomeruläre Filtrationsrate, CSA Ciclosporin A, TAC Tacrolimus

CoV-2 in unterschiedlichen Organen nachweisbar ist. Eine infizierte Niere könnte daher als virales Reservoir fungieren. Zwar ist das Risiko einer Übertragung von SARS-CoV-2 durch Organspende gering, dennoch sollten potenzielle Nierenspender auf SARSCoV-2 untersucht werden. Darüber hinaus sollten Lebendspender, die Sym- giger Prädiktor für die Mortalität. Daher fällt der interdisziplinären Betreuung und nephrologischen Nachsorge genesener COVID-19-Patienten eine entscheidende Bedeutung für das langfristige $\mathrm{Pa}$ tienten-Outcome zu.

\section{Medikamentöse Therapie der SARS-CoV-2 Infektion}

Zahlreiche antivirale und andere Wirkstoffe sind, basierend auf theoretischen Überlegungen, Fallberichten und In-vitro-Daten zu SARS und MERS, bei Patienten zum Einsatz gekommen.

Für eine spezifische antivirale Therapie liegen bislang noch keine ausreichenden Daten vor. Mit einer Reihe von Kandidatensubstanzen laufen klinische Studien, und einige Arzneimittel werden „off label“ oder im Rahmen individueller Heilversuche eingesetzt. Am weitesten verbreitet in der Anwendung scheinen Remdesivir (ein Nukleotidanalogon, primär entwickelt für die Behandlung von Infektionen mit Ebola- und Nipahviren) und Lopinavir/Ritonavir (Kaletra ${ }^{\circledR}$ ) in Kombination mit Interferon oder Chloroquin.

Die fortlaufend aktualisierte Tabelle der Arzneimittelkommission der deutschen Ärzteschaft (AkdÄ) hilft klinisch tätigen Ärzten, den Überblick über die Vielzahlmedikamentöser Therapieansätze zu behalten. Die Universität Liverpool hat eine Aufstellung wahrscheinlicher pharmakokinetischer Interaktionen von experimentellen von COVID19-Therapien veröffentlicht.

\section{Fazit für die Praxis}

- Erfreulicherweise sind mittlerweile die Infektionszahlen in Deutschland rückläufig, und die angesichts der Coronakrise getroffenen Schutzmaßnahmen werden schrittweise gelockert.

- Dennoch stellt die COVID-19(„,Coronavirus disease $2019^{\prime \prime}$ )-Pandemie weiterhin eine globale Bedrohung dar.

- Sichere und effektive Impfstoffe gelten als der Schlüssel für die Bekämpfung dieser Pandemie. 


\section{- Aktuell befinden sich laut Weltge- sundheitsorganisation (WHO) mehr als 130 SARS-CoV-2(,severe acute respiratory syndrome coronavi- rus $2^{\prime \prime}$ )-Impfstoff-Kandidaten in der Entwicklung, die meisten davon in der präklinischen bzw. explorativen Entwicklungsphase. \\ - In den kommenden Wochen und Monaten muss im Hinblick auf eine mögliche zweite COVID-19-Wel- le weiterhin eine auf Selbstschutz und Solidarität basierende und ziel- gruppenorientierte Risikostrategie verfolgt werden.}

\section{Korrespondenzadresse

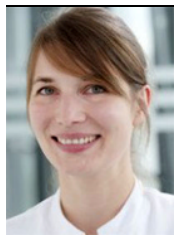 \\ Dr. H. Rohn \\ Klinik für Infektiologie, Westdeutsches Zentrum für Infektiologie (WZI), Universitätsmedizin Essen, Universität Duisburg-Essen Hufelandstr. 55, 45147 Essen, Deutschland Hana.Rohn@uk-essen.de}

Danksagung. Oliver Witzke wird durch die Rudolf Ackermann Stiftung (Stiftung für Klinische Infektiologie) unterstützt.

\section{Einhaltung ethischer Richtlinien}

Interessenkonflikt. 0 . Witzke erhielt Forschungsstipendien für klinische Studien, Referentenhonorare und Reisekosten von Amgen, Alexion, Astellas, Basilea, Biotest, Bristol-Myers Squibb, Correvio, Chiesi, Gilead, Hexal, Janssen, Dr. F. Köhler Chemie, MSD, Novartis, Roche, Pfizer, Sanofi, TEVA and UCB. H. Rohn und T. Feldkamp geben an, dass kein Interessenkonflikt besteht.

Für diesen Beitrag wurden von den Autoren keine Studien an Menschen oder Tieren durchgeführt. Für die aufgeführten Studien gelten die jeweils dort angegebenen ethischen Richtlinien.

\section{Literatur}

1. Sun $P$ et al (2020) Understanding of COVID19 based on current evidence. J Med Virol 92(6):548-551

2. Guan WJ et al (2020) Clinical characteristics of coronavirus disease 2019 in China. N Engl J Med 382(18):1708-1720

3. Wu Z, McGoogan JM (2020) Characteristics of and important lessons from the coronavirus disease 2019 (COVID-19) outbreak in China: summary of a report of 72314 cases from the Chinese center for disease control and prevention. JAMA 323(13):1239
4. Xu J et al (2020) Computed tomographic imaging of 3 patients with coronavirus disease 2019 pneumonia with negative virus real-time reversetranscription polymerase chain reaction test. Clin InfectDis. https://doi.org/10.1093/cid/ciaa207

5. Xie X et al (2020) Chest CT for typical 2019ncoV pneumonia: relationship to negative RT-PCR testing. Radiology. https://doi.org/10.1148/radiol. 2020200343

6. Perera RA et al (2020) Serological assays for severe acute respiratory syndrome coronavirus 2 (SARSCoV-2), March 2020. Euro Surveill. https://doi.org/ 10.2807/1560-7917.ES.2020.25.16.2000421

7. Lv H et al (2020) Cross-reactive antibody response between SARS-CoV-2 and SARS-CoV infections (bioRxiv)

8. To KK et al (2020) Temporal profiles of viral load in posterior oropharyngeal saliva samples and serum antibody responses during infection by SARS-CoV2: an observational cohort study. Lancet Infect Dis 20(5):565-574

9. Li T et al (2006) Long-term persistence of robust antibody and cytotoxic $T$ cell responses in recovered patients infected with SARS coronavirus. Plos One 1:e24

10. Payne DC et al (2016) Persistence of antibodies against middle east respiratory syndrome coronavirus. Emerg Infect Dis 22(10):1824-1826

11. Zhou F et al (2020) Clinical course and risk factors for mortality of adult inpatients with COVID-19 in Wuhan, China: a retrospective cohort study. Lancet 395(10229):1054-1062

12. Hirsch JS etal (2020) Acute kidney injury in patients hospitalized with COVID-19. Kidney Int. https:// doi.org/10.1016/j.kint.2020.05.006

13. Cheng $Y$ et al (2020) Kidney disease is associated with in-hospital death of patients with COVID-19. Kidney Int 97(5):829-838

14. Chen T et al (2020) Clinical characteristics of 113 deceased patients with coronavirus disease 2019 retrospective study. BMJ 368:m1091

15. Martinez-Rojas MA, Vega-Vega O, Bobadilla NA (2020) Is the kidney a target of SARS-CoV-2? Am J Physiol Renal Physiol 318(6):F1454-F1462

16. Puelles VG et al (2020) Multiorgan and renal tropism of SARS-CoV-2. N Engl J Med. https://doi. org/10.1056/NEJMc2011400

17. Yao XH et al (2020) A pathological report of three COVID-19 cases by minimal invasive autopsies. Zhonghua Bing Li Xue Za Zhi 49(5):411-417

18. Wan $Y$ et al (2020) Receptor recognition by the novel coronavirus from Wuhan: an analysis based on decade-long structural studies of SARS coronavirus. J Virol. https://doi.org/10.1128/jvi. 00127-20

19. Hoffmann M et al (2020) SARS-CoV-2 cell entry depends on ACE2 and TMPRSS2 and is blocked by a clinically proven protease inhibitor. Cell 181(2):271-280.e8

20. Yan $R$ et al (2020) Structural basis for the recognition of SARS-CoV-2 by full-length human ACE2. Science 367(6485):1444-1448

21. Ye $M$ et al (2006) Glomerular localization and expression of angiotensin-converting enzyme 2 and angiotensin-converting enzyme: implications for albuminuria in diabetes. J Am Soc Nephrol 17(11):3067-3075

22. Remuzzi A, Remuzzi G (2020) COVID-19 and Italy: what next? Lancet 395(10231):1225-1228

23. Ishiyama $Y$ et al (2004) Upregulation of angiotensin-converting enzyme 2 after myocardial infarction by blockade of angiotensin II receptors. Hypertension 43(5):970-976
24. Fang L, Karakiulakis G, Roth M (2020) Are patients with hypertension and diabetes mellitus at increased risk for COVID-19 infection? Lancet RespirMed 8(4):e21

25. Reynolds HR et al (2020) Renin-angiotensinaldosterone system inhibitors and risk of Covid19. N Engl J Med. https://doi.org/10.1056/ nejmc2013707

26. Mancia Getal (2020) Renin-angiotensin-aldosterone system blockers and the risk of Covid-19. NEng JMed. https://doi.org/10.1056/NEJMoa2006923

27. Li J et al (2020) Association of renin-angiotensin system inhibitors with severity or risk of death in patients with hypertension hospitalized for coronavirus disease 2019 (COVID-19) infection in Wuhan, China. JAMA Cardiol. https://doi.org/10. 1001/jamacardio.2020.1624

28. de Abajo FJ et al (2020) Use of renin-angiotensinaldosterone system inhibitors and risk of COVID-19 requiring admission to hospital: a case-population study. Lancet 395(10238):1705-1714

29. Mackey K et al (2020) Risks and impact of angiotensin-converting enzyme inhibitors or angiotensin-receptor blockers on SARS-CoV-2 infection in adults. Ann Intern Med. https://doi. org/10.7326/M20-1515

30. Kuba K et al (2005) A crucial role of angiotensin converting enzyme 2 (ACE2) in SARS coronavirusinduced lung injury. Nat Med 11(8):875-879

31. Zhang P et al (2020) Association of inpatient use of angiotensin-converting enzyme inhibitors and angiotensin II receptor blockers with mortality among patients with hypertension hospitalized with COVID-19. CircRes 126(12):1671-1681

32. Yang $G$ et al (2020) Effects of angiotensin II receptor blockers and ACE (AngiotensinConverting Enzyme) inhibitors on virus infection, inflammatory status, and clinical outcomes in patients with COVID-19 and hypertension: a single-center retrospective study. Hypertension 76(1):51-58

33. Kliger AS, Silberzweig J (2020) Mitigating risk of COVID-19 in dialysis facilities. Clin J Am Soc Nephrol 15(5):707-709

34. Li HS et al (2018) Effect of interferon alpha and cyclosporine treatment separately and in combination on Middle East RespiratorySyndrome Coronavirus (MERS-CoV) replication in a human in-vitro and ex-vivo culture model. Antiviral Res 155:89-96 